\title{
Bilateral Cerebellar Infarcts from Vertebral Artery Insufficiency Caused by Cervical Osteophytes
}

\section{Ripul R Panchal*, Daniel S Hutton and Kee D Kim}

Department of Neurological Surgery, University of California-Davis Medical Center, Sacramento, California, USA

\begin{abstract}
Background: In previous reports, the patients are described to have transient symptoms from physiologic rotation or extension of the cervical spine, resulting from a cervical osteophyte compressing the vertebral artery and causing vertebral artery insufficiency, known as Bow Hunter syndrome.
\end{abstract}

Methods: An 85-year-old female presented with new onset occipital headaches, nausea, vomiting and vertigo that were not precipitated by change in head position. Patient had bilateral cerebellar infracts. Patient underwent decompression and instrumented stabilization of the cervical spine from the posterior approach.

Results: At one-year follow-up, patient remained stroke free with patent vertebral artery.

Conclusion: To our knowledge, this is the first report of bilateral infraction from a vertebral artery insufficiency caused by cervical osteophytes without history of transient symptoms from movement of the head or neck, a variant of the Bow Hunter syndrome. Anterior versus posterior approach for vertebral artery insufficiency from osteophytic compression should be primarily based on location of the pathology and not the cervical level of involvement.

Keywords: Bow hunter syndrome; Cervical osteophyte; Infarction; Vertebral artery; Surgery; Vertebral artery insufficiency

Abbreviations: VAI: Vertebral Artery Insufficiency; VA: Vertebral Artery; CT: Computed Tomography; MRI: Magnetic Resonance Imaging; CTA: Computed Tomographic Angiography

\section{Introduction}

Vertebral Artery Insufficiency (VAI) or Bow Hunter syndrome result from a cervical osteophyte compressing the Vertebral Artery (VA) is a rare condition resulting in transient symptoms from physiologic rotation or extension of the cervical spine [1-15]. Presenting symptoms include history of dizziness, vertigo, syncope, nausea, or sensorimotor disturbance $[3,9,16]$. With the change in cervical position, especially rotation or extension, the flow in the VA may be compromised leading to transient ischemia in the supplying tissues when the contralateral VA is hypoplastic or congenitally atretic [1,17-19]. In previous cases reported, patients present with transient symptoms with certain neck position occurring for duration of time $[5,9,14,16]$. To our knowledge, this is the first report of bilateral infarction from a vertebral artery insufficiency caused by cervical osteophytes without history of neck motion-related transient symptoms, a variant of Bow Hunter syndrome.

\section{Case Report}

An 85-year-old female presented with new onset of occipital headaches, nausea, vomiting and vertigo that were not precipitated by change in head or neck position. Patient had a slightly unsteady gait but otherwise neurological exam was normal. There was no change in symptoms observed with change in neck position. Initial head Computed Tomography (CT) scan and followed by brain Magnetic Resonance Imaging (MRI) showed acute left cerebellar infarction within the posterior inferior cerebellar artery distribution and hemorrhagic infarction within the right cerebellar hemisphere (Figure 1). Cranial and cervical Computed Tomographic Angiography (CTA) revealed an osteophytic compression of the left VA at C4-5 with a small intraluminal thrombus just proximal to this area (Figure 2, 3A, 3B and $3 \mathrm{C})$.

The left acute cerebellar and right sub-acute paramedian cerebellar infarcts were believed to originate from left VAI whose distribution may extend over to the right cerebellar hemisphere. It was concluded that the insufficiency of the left VA was due to the osteophytic spurs. Immediately, the patient was started on aspirin and clopidogrel (Plavix, Sanofi-aventis, Bridge water, New Jersey). However, due to the lack of the rotational component of Bow Hunter's syndrome with transient symptoms in patient's presentation, and non-dynamic radiographic studies illustrating significant stenosis, patient was considered at high risk and surgical intervention to decompress the left VA was planned.

A midline posterior approach was performed for multilevel decompression of the VA stenosis and stabilization of the cervical spine with instrumentation (Figure 4). Complete resection of the lateral mass

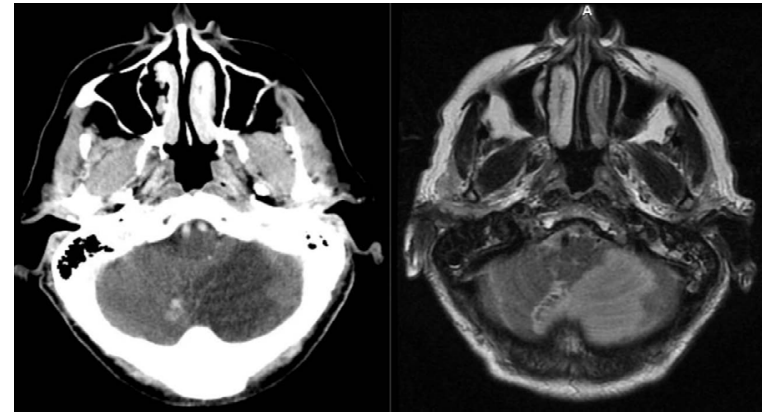

Figure 1: Axial CT (left) and Brain MR images (right) showing bilateral cerebellar infarcts.

*Corresponding author: Ripul R Panchal, Department of Neurological Surgery, University of California, Davis School of Medicine, 4860 Y Street, Suite 3740 Sacramento, CA 95817, USA, Tel: 916-734-3658; Fax: 916-452-2580; E-mail: ripul. panchal@ucdmc.ucdavis.edu

Received June 20, 2012; Accepted July 25, 2012; Published July 27, 2012

Citation: Panchal RR, Hutton DS, Kim KD (2012) Bilateral Cerebellar Infarcts from Vertebral Artery Insufficiency Caused by Cervical Osteophytes. J Spine 1:122 doi:10.4172/2165-7939.1000122

Copyright: (c) 2012 Panchal RR, et al. This is an open-access article distributed under the terms of the Creative Commons Attribution License, which permits unrestricted use, distribution, and reproduction in any medium, provided the original author and source are credited. 
of left C4 and C5 and partial resection of C3 and C6 was performed. Intraoperative microdoppler was utilized to monitor the location of the VA and its flow.

Postoperatively, the imaging demonstrated complete decompression of the left VA (Figure 3D, 3E and 3F) and the hospital course was uneventful. At one-year follow up after surgery, left VA remained patent and regained its normal caliber (Figure 3G, 3H and 3I). She was stroke-free and asymptomatic having completely recovered from her stroke.

\section{Discussion}

Hutchinson and Yates's anatomical studies first described the compromise of second segment of the VA from spondylotic etiology [20]. In the subaxial cervical spine, the formation of osteophytic spurs is most common at the C5 and C6 (56\%). [10] In contrast to other subaxial levels, a fibrous band tethers the VA as the second segment of VA enters the C6 transverse foramen. With repetitive motion over time, osteophytic spur formation may result in development of symptomatic VA stenosis [5,10]. Rotational stenosis of the VA may

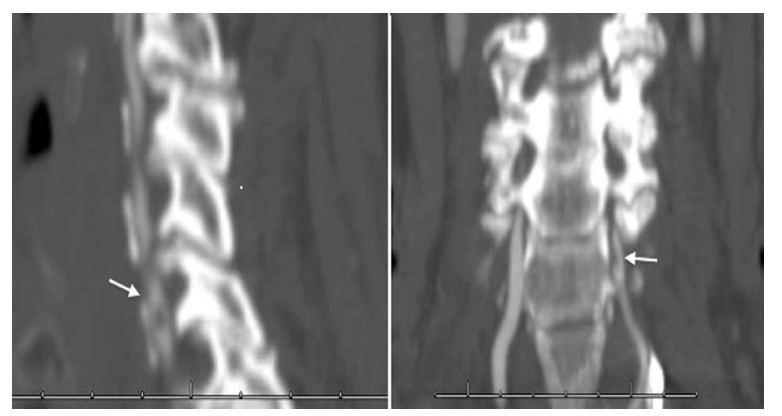

Figure 2: Axial CTA scans of left vertebral artery. Lateral (left) and coronal (right) CTA scans showing small intraluminal thrombus at C-6 (arrows).

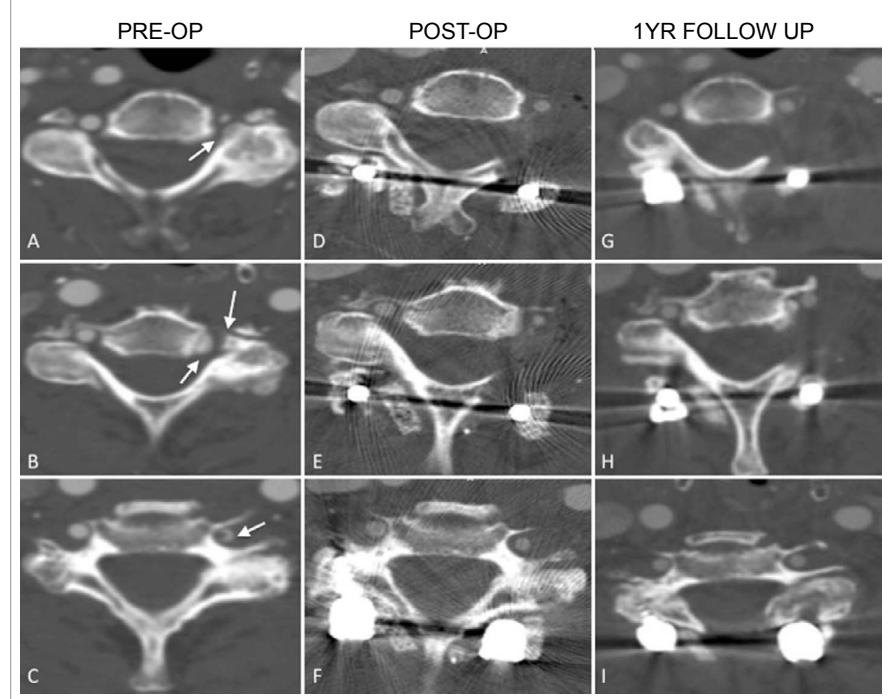

Figure 3: $(\mathrm{a}$ and $\mathrm{b})$ Preoperative images at C4 and C5 showing osteophytes compromising the flow of the left vertebral artery (arrows). (c) Preoperative image showing intraluminal thrombus (arrow). (d-f) Postoperative images at $\mathrm{C} 4, \mathrm{C} 5$, and $\mathrm{C} 6$ respectively showing slight increase in the caliber of left vertebral artery. (g-i) One-year follow-up images at C4, C5, and C6 respectively, demonstrating left vertebral artery caliber to be similar to the right vertebral artery.

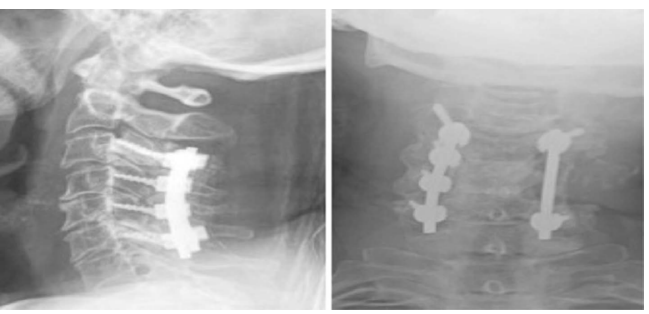

Figure 4: Lateral (left) and antero-posterior radiographs (right) obtained immediately following C3-6 posterior fixation and fusion using lateral mass screws.

result anteromedially or posteriorly from osteophytes arising from uncinate process or facet complex, respectively $[5,9,12,21]$. Our patient had combination of both anteriomedial and posterior osteophytic spur formation (Figure 3B). Due to the lack of the rotational component of Bow Hunter's syndrome with transient symptoms in our patient's presentation, and nondynamic radiographic studies illustrating significant stenosis, we describe this as a Bow Hunter syndrome variant.

$\mathrm{Lu}$ et al. [16] recommended anterior approach for lesions at or caudal to $\mathrm{C} 4$ and posterior approach for lesions rostral to $\mathrm{C} 4$. However, Citow and MacDonald [9] and Petridis et al. [22] utilized posterior approaches with good results for decompression at C6 and C4-5, respectively. We used posterior approach because the VA was compromised from the combination of both uncinate process and facet complex, and multiple levels were involved. Since the patient had complete resection of the lateral mass of left $\mathrm{C} 4$ and $\mathrm{C} 5$, instrumented stabilization was performed (Figure 3D, 3E and 3F) At one-year followup after surgery, the patient is stroke-free while the imaging revealed the cervical spine to be stable, and that the left VA regained its normal caliber (Figure 3G, 3H and 3I). Anterior versus posterior approach for VAI from osteophytic compression should be primarily based on location of the pathology and not the cervical level of involvement.

\section{Acknowledgement}

We like to thank Dr. Piero Verro for his contribution.

\section{References}

1. Anson JA, Spetzler RF (1995) Surgery for vertebrobasilar insufficiency: extracranial. New York: McGraw-Hill.

2. Bakay L, Leslie EV (1965) Surgical treatment of vertebral artery insufficiency caused by cervical spondylosis. J Neurosurg 23: 596-602.

3. George B, Laurian C (1989) Impairment of vertebral artery flow caused by extrinsic lesions. Neurosurgery 24: 206-214.

4. Gortvai $P$ (1964) Insufficiency of Vertebral Artery Treated by Decompression of Its Cervical Part. Br Med J 2: 233-234

5. Miele VJ, France JC, Rosen CL (2008) Subaxial positional vertebral artery occlusion corrected by decompression and fusion. Spine (Phila Pa 1976) 33 E366-370.

6. Hardin CA, Williamson WP, Steegmann AT (1960) Vertebral artery insufficiency produced by cervical osteoarthritic spurs. Neurology 10: 855-858.

7. Hardin CA (1965) Vertebral Artery Insufficiency Produced by Cervical Osteoarthritic Spurs. Arch Surg 90: 629-633.

8. Keggi KJ, Granger DP, Southwick WO (1966) Vertebral artery insufficiency secondary to trauma and osteoarthritis of the cervical spine. Yale $\mathrm{J}$ Biol Med 38: 471-478.

9. Citow JS, Macdonald RL (1999) Posterior decompression of the vertebral artery narrowed by cervical osteophyte: case report. Surg Neurol 51: 495-498.

10. Nagashima C (1970) Surgical treatment of vertebral artery insufficiency caused by cervical spondylosis. J Neurosurg 32: 512-521. 
Citation: Panchal RR, Hutton DS, Kim KD (2012) Bilateral Cerebellar Infarcts from Vertebral Artery Insufficiency Caused by Cervical Osteophytes. J Spine 1:122. doi:10.4172/2165-7939.1000122

11. Powers SR Jr., Drislane TM, Nevins S (1961) Intermittent vertebral artery compression; a new syndrome. Surgery 49: 257-264.

12. Sheehan S, Bauer RB, Meyer JS (1960) Vertebral artery compression in cervical spondylosis, angiographic demonstration during life of vertebral artery insufficiency due to rotation and extension of the neck. Neurology 10: 386-396.

13. Smith DR, Vanderark GD, Kempe LG (1971) Cervical spondylosis causing vertebrobasilar insufficiency: a surgical treatment. J Neurol Neurosurg Psychiatry 34: 388-392

14. Spetzler RF, Hadley MN, Martin NA, Hopkins LN, Carter LP, et al. (1987) Vertebrobasilar insufficiency. Part 1: Microsurgical treatment of extracranial vertebrobasilar disease. J Neurosurg 66: 648-661.

15. Sullivan HG, Harbison JW, Vines FS, Becker D (1975) Embolic posterior cerebral artery occlusion secondary to spondylitic vertebral artery compression. Case report. J Neurosurg 43: 618-622.

16. Lu DC, Zador Z, Mummaneni PV, Lawton MT (2010) Rotational vertebral artery occlusion-series of 9 cases. Neurosurgery 67: 1066-1072.
17. Kuther TA, Nesbit GM, Clark WM, Barnwell SL (1997) Rotational vertebral artery occlusion: a mechanism of vertebrobasilary insufficiency. Neurosurgery 41: 427-433.

18. Strupp M, Planck JH, Arbusow V, Steiger HJ, Bruckmann H, et al. (2000) Rotational vertebral artery occlusion syndrome with vertigo due to "labyrinthine excitation”. Neurology 54: 1376-1379.

19. Sturzenegger M (1994) Headache and neck pain: the warning symptoms of vertebral artery dissection. Headache 34: 187-193.

20. Hutchinson EC, Yates PO (1956) The cervical portion of the vertebral artery $A$ clinico-pathological study. Brain 79: 319-331.

21. Ebraheim NA, Lu J, Biyani A, Brown JA, Yeasting RA (1997) Anatomic considerations for uncovertebral involvement in cervical spondylosis. Clin Orthop Relat Res: 200-206.

22. Petridis AK, Barth H, Buhl R, Mehdorn HM (2008) Vertebral artery decompression in a patient with rotational occlusion. Acta Neurochir (Wien) 150: 391-394. 